\title{
ResearchArticle
}

\section{Studies on fungicides seed treatment against damping- off in seedling of rough lemon (Citrus jambhiri Lush) in Nagpur Mandarin Nursery}

\author{
Bhupendra Thakre and Uttam Soni
}

\begin{abstract}
SUMMARY
Mandarin seedlings are very much susceptible to damping off disease. In most of the farmers' nursery mandarin seedlings suffer from pre-emergence and post emergence damping off causing seedling decline and is a great threat for raising seedling. Experiments were conducted in Jawaharlal Nehru Krishi Vishwa Vidyalaya, Zonal Agriculture Research Station under Technology Mission on citrus project during 2015-16. Completely Randomize Design (RBD), replicated thrice with ten treatments including control. Six fungicides viz., carbendazim WP $50 \%$, fostel WP $80 \%$, copper Oxy Chloride WP 50\%, Carbendazim 12\% WP+ Mancozeb 63\% WP, Captan 50\% WP, metalaxyl 04\% WP+ mancozeb 63\% WP were selected for these experiment studies. Before sowing seeds were treated with these selected fungicides. Untreated seed served as control.Damping off in rough lemon seedling of citrus nursery could easily be reduced by fungicide seed treatment by carbendazim WP 50\% and indicate that carbendazim WP $50 \%$ increased maximum per cent in shoot length $(13.37 \mathrm{~cm} /$ $74.47 \%)$, root length $(2.45 \mathrm{~cm} / 67.57 \%)$ and dry weight $(52.74 \%)$ over control.
\end{abstract}

Key Words : Citrus, Rough lemon, Damping off, Pythium sp., Seed treatment, Fungicides

How to cite this article : Thakre, Bhupendra and Soni, Uttam (2018). Studies on fungicides seed treatment against damping-off in seedling of rough lemon (Citrus jambhiri Lush) in Nagpur Mandarin Nursery. Internat. J. Plant Sci., 13 (1): 153-157, DOI: 10.15740/ HAS/IJPS/13.1/153-157.

Article chronicle : Received : 14.11.2017; Revised : 03.12.2017; Accepted : 17.12.2017

Email : bhupendra_thakrejnkvv@rediffmail.com

Address of the Co-authors:

Uttam Soni, Zonal Agricultural Research Station (JNKVV), Chhindwara (M.P.) India 